\title{
Molecular prevalence of Dirofilaria immitis and Wolbachia infections in pet and semi-domesticated cats in Bangkok, Thailand
}

\author{
Naris Thengchaisri ${ }^{1,2}\left(\mathbb{D}\right.$, Tawin Inpankaew ${ }^{(\mathbb{D}}$, Surapong Arthitwong ${ }^{4}$, Jörg M. Steiner ${ }^{(\mathbb{D})}$ and Panpicha Sattasathuchana ${ }^{1}$ (D) \\ 1. Department of Companion Animal Clinical Sciences, Faculty of Veterinary Medicine, Kasetsart University, Bangkok, \\ Thailand; 2. Tippimarn Veterinary Hospital, Chulabhorn Royal Academy, 906/1 Pong Ta Long Subdistrict, Pak Chong \\ district, Nakhon Ratchasima, 30130, Thailand; 3. Department of Parasitology, Faculty of Veterinary Medicine, Kasetsart \\ University, Bangkok, Thailand; 4. Department of Anatomy, Faculty of Veterinary Medicine, Kasetsart University, Bangkok, \\ Thailand; 5. Department of Small Animal Clinical Sciences, Gastrointestinal Laboratory, College of Veterinary Medicine \\ and Biomedical Sciences, Texas A\&M University, College Station, Texas, USA. \\ Corresponding author: Panpicha Sattasathuchana, e-mail: psatta99@gmail.com \\ Co-authors: NT: ajnaris@yahoo.com, TI: tawin.i@ku.th, SA: fvetsra@ku.ac.th, JMS: jsteiner@cvm.tamu.edu \\ Received: 28-09-2021, Accepted: 03-01-2022, Published online: 03-02-2022
}

doi: www.doi.org/10.14202/vetworld.2022.239-243 How to cite this article: Thengchaisri N, Inpankaew T, Arthitwong S, Steiner JM, Sattasathuchana P (2022) Molecular prevalence of Dirofilaria immitis and Wolbachia infections in pet and semi-domesticated cats in Bangkok, Thailand, Veterinary World, 15(2): 239-243.

\begin{abstract}
Background and Aim: Although cats are not natural hosts for heartworm infections (Dirofilaria immitis), evidence suggests that feline heartworm disease can be detrimental because of a severe inflammatory response. Recent studies have found that infection with bacteria of the genus Wolbachia is the principal cause of acute inflammatory filaria disease; nonetheless, the prevalence of cats naturally infected with heartworms and Wolbachia remains unclear. This study aimed to estimate the prevalence and current distribution of feline heartworm disease and its association with Wolbachia infection in pet and semidomesticated cats in Bangkok, Thailand.
\end{abstract}

Materials and Methods: A total of 260 cats (130 pet cats and 130 semi-domesticated cats) were enrolled in this study. Blood samples were placed into ethylenediaminetetraacetic acid tubes for hematological analysis and DNA extraction. A polymerase chain reaction (PCR) was performed to analyze samples for the presence of D. immitis and Wolbachia infections.

Results: The prevalence (95\% confidence interval [CI]) of D. immitis infection in pet, semi-domesticated, and all cats were $3.9 \%(1.3-8.8 \%), 27.7 \%(20.2-36.2 \%)$, and 19.6\% (15.0-25.0\%), respectively. The prevalence (95\% CI) of Wolbachia infection in pet, semi-domesticated, and all cats were 18.5\% (12.2-26.2\%), 31.5\% (23.7-40.3\%), and 25.0\% (19.9-30.7\%), respectively. The prevalence of $D$. immitis and Wolbachia infections in semi-domesticated cats was significantly higher than in pet cats $(\mathrm{p}=0.002$ and $\mathrm{p}=0.022$, respectively). There was a significant association between $D$. immitis and Wolbachia infections $(\mathrm{p}<0.001)$. There was also a significant association between $D$. immitis infection and the presence of eosinophilia $(\mathrm{p}<0.045)$.

Conclusion: From the PCR analysis, it can be concluded that semi-domesticated cats were at higher risk for D. immitis infection than pet cats. There was a significant association between positive $D$. immitis infection and positive Wolbachia infection. Combinations of anthelmintic and antimicrobial therapy should be considered in heartworm-positive cats.

Keywords: cat, heartworm, prevalence, Wolbachia.

\section{Introduction}

Dirofilaria immitis is a vector-borne parasite that causes cardiopulmonary diseases in dogs and cats worldwide [1-8]. Dogs are a major reservoir for $D$. immitis, but cats and humans have also been reported as incidental hosts [7]. Cats are considered naturally resistant hosts because of the high mortality rate of L5 larvae in the pulmonary arteries of cats $[4,9]$. In Thailand, the prevalence of $D$. immitis in pet and semi-domesticated cats has been reported as $4.6 \%$ and $36.4 \%$, respectively $[3,6]$. Heartworm disease can lead to eosinophilic pneumonitis and severe

Copyright: Thengchaisri, et al. Open Access. This article is distributed under the terms of the Creative Commons Attribution 4.0 International License (http://creativecommons.org/licenses/ by/4.0/), which permits unrestricted use, distribution, and reproduction in any medium, provided you give appropriate credit to the original author(s) and the source, provide a link to the Creative Commons license, and indicate if changes were made. The Creative Commons Public Domain Dedication waiver (http:// creativecommons.org/publicdomain/zero/1.0/) applies to the data made available in this article, unless otherwise stated. pulmonary thromboembolism [10]. Heartwormassociated respiratory disease has been linked with the release of the endosymbiotic bacterium from filarial nematodes [11]. Wolbachia endosymbiont is a Gram-negative, intracellular bacterium harbored in all life stages of parasites [12]. Wolbachia organisms have been reported in filarial nematodes, such as Brugia malayi and D. immitis [13,14]. Wolbachia surface proteins and antibodies are released when the parasite dies $[12,14]$. Although the pathogenetic role of Wolbachia in mammalian host cells is not clearly identified, Wolbachia antigens and antibodies have been known to stimulate the inflammation of mammalian host cells $[14,15]$.

Several reports have identified the prevalence of D. immitis in dogs. The prevalence of Wolbachia infection among cats in Italy and dogs in Thailand has been reported at $10.4 \%$ and $82.5 \%$, respectively $[16,17]$. To the best of our knowledge, there has beenno report concerning the prevalence of Wolbachia infection 
among cats in Thailand. In addition, there are limited reports of the prevalence of and the association between $D$. immitis and Wolbachia infection in cats. Cats are often raised in households as human companions; thus, zoonotic diseases from cats may be transmitted to humans. D. immitis and Wolbachia endosymbiont infections have been reported in humans $[18,19]$.

The aims of this study were 3-fold: (1) Estimate the prevalence of D. immitis and Wolbachia endosymbiont infections; (2) evaluate the association between D. immitis and Wolbachia infections in pet and semi-domesticated cats; and (3) evaluate the association between $D$. immitis infection and hematological parameters.

\section{Materials and Methods}

\section{Ethical approval and informed consent}

All sample collection protocols were reviewed and approved by the Kasetsart University Institutional Animal Care and Use Committee (approval number \#ACKU60-VET-032). The study adhered to the ARRIVE (Animal Research: Reporting of In Vivo Experiments) guidelines. All cat owners were informed about the study goals, their rights, participant requirements, and permission to collect a blood sample from their cat. Written informed consent for semi-domesticated cats and client-owned cats was obtained from the head of the community or the cat's owner, respectively.

\section{Study period and location}

The domesticated cat samples were collected from cats visiting Kasetsart University Veterinary Teaching Hospital, Bangkok, Thailand, for an annual check-up between July 2017 to June 2018. The semi-domesticated cats samples were collected from cats living in the Thai temples in Bangkok, Thailand, from March 2017 to June 2017. The samples were processed at Faculty of Veterinary Medicine, Kasetsart University, Bangkhen campus.

\section{Sample collection}

A total of 260 cats were enrolled in the study: 130 pet cats and 130 semi-domesticated cats. A complete history was collected for all domesticated cats, and a physical examination was performed to enroll only clinically healthy cats with a history of routine flea control and deworming programs. A whole-blood sample of approximately $3 \mathrm{~mL}$ volume was collected by a licensed veterinarian through either a cephalic or jugular puncture and was placed into an ethylenediaminetetraacetic acid (EDTA)-anticoagulated blood tube. A complete blood count was immediately performed using an automated hematology analyzer (Abbott CELL-DYN 3700 Hematology Analyzer, Abbott, Germany). The EDTA blood samples were stored at $-20^{\circ} \mathrm{C}$ before DNA extraction.

\section{DNA extraction}

DNA was extracted from $250 \mu \mathrm{L}$ of EDTAanticoagulated blood using a commercial DNA extraction kit (E.Z.N.A. ${ }^{\circledR}$ Blood DNA Mini Kit, Omega Bio-Tek Inc., Norcross, GA, USA) according to the manufacturer's instructions. Final elution of the DNA was made in $100 \mu \mathrm{L}$ of Tris-EDTA buffer (10 $\mathrm{mM}$ Tris-Cl, $0.5 \mathrm{mM}$ EDTA, $\mathrm{pH}$ 9.0). The extracted DNA was stored at $-20^{\circ} \mathrm{C}$ until used for the polymerase chain reaction (PCR) assays.

\section{PCR analysis and sequencing}

All extracted DNAs were subject to PCR assays as previously described [20] to detect $D$. immitis infection based on the cytochrome $\mathrm{C}$ oxidase subunit I (COI) gene. Wolbachia infection was detected based on the Wolbachia protein-coding housekeeping ftsZ gene [16], as shown in Table-1 [16,20]. The PCR product was analyzed using $1.5 \%$ agarose gel electrophoresis. All positive PCR products were purified using the FavorPrep ${ }^{\text {TM }}$ GEL/PCR Purification Kit (Favorgen Biotech Corporation, Pin-Tung, Taiwan) according to the manufacturer's protocol and submitted to Macrogen (Macrogen Inc., Seoul, Korea) for DNA sequencing. All sequences were accumulated using Finch TV 1.4.0 (Geospiza Inc., Seattle, WA, USA) and compared with known sequences from the GenBank ${ }^{\mathrm{TM}}$ database (National Center for Biotechnology Information, Bethesda, MD, USA) using the BLAST (Basic Local Alignment Search Tool) algorithm (http://blast.ncbi. nlm.nih.gov/Blast.cgi).

\section{Statistical analysis}

All analyses were performed using commercially available statistical software packages (JMP version JMP Pro 10, SAS institute, Cary, NC, USA; GraphPad Prism version 5.0, Graph-Pad Software, LAJolla, CA, USA; and STATA version 14.2 StataCorp LLC, College Station, TX, USA), with $\mathrm{p}<0.05$ defined as statistically significant. The prevalence and $95 \%$ confidence interval (CI) of D. immitis and Wolbachia infections in pet and semi-domesticated cats were determined

Table-1: Oligonucleotide primers used to detect $D$. immitis and Wolbachia endosymbiont in blood samples from cats.

\begin{tabular}{|c|c|c|c|c|}
\hline Pathogen & $\begin{array}{l}\text { Gene target } \\
\text { (size }[\mathrm{bp}])\end{array}$ & Oligonucleotide sequence & $\begin{array}{l}\text { Annealing } \\
\text { temperature }\end{array}$ & Reference \\
\hline D. immitis & COI (200) & $\begin{array}{l}\text { DI COI-F1 (5'- AGTGTAGAGGGTCAGCCTGAGTTA-3') } \\
\text { DI COI-R1 (5'- ACAGGCACTGACAATACCAAT-3') }\end{array}$ & $59^{\circ} \mathrm{C}$ & {$[20]$} \\
\hline $\begin{array}{l}\text { Wolbachia } \\
\text { endosymbiont }\end{array}$ & FtsZ (147) & $\begin{array}{l}\text { Wol1-fwd (5'-CCTGTACTATATCCAAGAATTACTG-3') } \\
\text { Wol1-R (5'-ACTATCCTTATATGTTCCATAATTTC-3') } \\
\text { Wol7-fwd (5'-GGTGGAAATGCTGTGAATAAC-3') } \\
\text { Wol7-R (5'-AGCACCGAGCCCTTAA-3') }\end{array}$ & $\begin{array}{l}51^{\circ} \mathrm{C} \\
57^{\circ} \mathrm{C}\end{array}$ & {$[16]$} \\
\hline
\end{tabular}

D. immitis=Dirofilaria immitis, $\mathrm{COI}=$ Cytochrome $\mathrm{C}$ oxidase subunit I 
using cross-tabulations. The prevalence of $D$. immitis and Wolbachia infections in pet and semi-domesticated cats was compared using Fisher's exact test. Fisher's exact test was also used to determine the association between D. immitis and Wolbachia infections, between $D$. immitis infection and hematological parameters, and between Wolbachia infection and hematological parameters.

\section{Results}

The mean age \pm standard deviation (SD) and the age range of all cats were $4.1 \pm 2.9$ years and 0.3 15 years, respectively. The mean age $\pm \mathrm{SD}$ and the minimum and maximum ages of the semi-domesticated cats were $2.8 \pm 2.0$ years and $0.83-13$ years, respectively. The sex distribution of pet and semi-domesticated cats was 62 males versus 68 females and 47 males versus 83 females. All semi-domesticated cats were domestic shorthair cats, whereas the pet cats consisted of 67 shorthair and 63 longhair cats. The nucleotide sequences of the partial COI gene for which the filarial nematodes were able to be classified was $98-100 \%$ identical with $D$. immitis (GenBank: MK250757). The sequences of the ftsZ gene for Wolbachia identified in cats were 100\% identical to the Wolbachia endosymbiont of D. immitis (GenBank: AJ495000).

The prevalence $(95 \% \mathrm{CI})$ of $D$. immitis infection in pet, semi-domesticated, and all cats was 3.9\% (1.3-8.8\%), 27.7\% (20.2-36.2\%), and 19.6\% (15.0$25.0 \%$ ), respectively (Table-2). The prevalence of $D$. immitis infection in semi-domesticated cats was significantly higher than in pet cats $(\mathrm{p}=0.002$; Table-2). The prevalence $(95 \% \mathrm{CI})$ of Wolbachia infection in pet, semi-domesticated, and all cats was $18.5 \%$ (12.2-26.2\%), 31.5\% (23.7-40.3\%), and 25.0\% (19.9$30.7 \%$ ), respectively (Table-2).
The prevalence of Wolbachia infection in semi-domesticated cats was significantly higher than in pet cats $(\mathrm{p}=0.022$; Table-2). There was an association between D. immitis and Wolbachia infections $(\mathrm{p}<0.001$; Table-3).

There was no association between $D$. immitis infection and the presence of anemia $(\mathrm{p}=0.718)$ or leukocytosis ( $\mathrm{p}=0.278$; Table-4). However, there was a significant association between $D$. immitis infection and the presence of eosinophilia $(\mathrm{p}=0.045$; Table- 4$)$. There was no association between Wolbachia infection and the presence of anemia $(\mathrm{p}=0.764)$, leukocytosis $(\mathrm{p}=0.364)$, or eosinophilia $(\mathrm{p}=0.089$; Table-5).

\section{Discussion}

Even though dogs are a natural host for heartworm infection, cats and other mammal species can also be affected. The present study found that the overall prevalence of $D$. immitis infection among cats in Bangkok was $19.6 \%$. Although more than a quarter of semi-domesticated cats $(27.7 \%)$ were affected with $D$. immitis infection, the prevalence of $D$. immitis infection in pet cats $(3.9 \%)$ was significantly lower. The overall Wolbachia endosymbiont infection prevalence in Bangkok cats was $25.0 \%$. The prevalence of Wolbachia endosymbiont infection in semi-domesticated cats $(31.5 \%)$ was also significantly higher than in pet cats (18.5\%). Cats with positive D. immitis infection had a significantly higher risk of having Wolbachia infection than cats negative for $D$. immitis infection. There was also an association between $D$. immitis infection and the presence of eosinophilia, suggesting the presence of host response to heartworm infection in cats.

Detection of the adult female $D$. immitis antigen is commonly used to detect heartworm infection in dogs. However, a false-negative $D$. immitis antigen test is more common in cats than dogs because cats

Table-2: Summary of D. immitis and Wolbachia endosymbiont infections in pet and semi-domesticated cats.

\begin{tabular}{|c|c|c|c|c|c|}
\hline Diagnosis & Total (n) & Positive (n) & Negative ( $\mathrm{n}$ ) & Prevalence (\%; CI) & p-value \\
\hline \multicolumn{6}{|l|}{ D. immitis } \\
\hline Pet cats & 130 & 15 & 115 & $3.85(1.26-8.75)$ & \\
\hline Semi-domesticated cats & 130 & 36 & 94 & $27.69(20.21-36.22)$ & \\
\hline Total cats & 260 & 51 & 209 & $19.62(14.97-24.97)$ & 0.002 \\
\hline \multicolumn{6}{|l|}{ Wolbachia endosymbiont } \\
\hline Pet cats & 130 & 24 & 106 & $18.46(12.20-26.21)$ & \\
\hline Semi-domesticated cats & 130 & 41 & 89 & $31.54(23.67-40.27)$ & \\
\hline Total cats & 260 & 65 & 195 & $25.00(19.86-30.72)$ & 0.022 \\
\hline
\end{tabular}

D. immitis=Dirofilaria immitis, $\mathrm{CI}=$ Confidence interval

Table-3: Association between D. immitis and Wolbachia endosymbiont infections in all cats.

\begin{tabular}{lcccc}
\hline D. immitis infection & \multicolumn{2}{c}{ Wolbachia endosymbiont infection } & p-value \\
\cline { 2 - 4 } & Positive (n) & Negative (n) & Total & \\
\hline Positive & 51 & 0 & 51 & 209 \\
Negative & 14 & 195 & 260 & $<0.001$ \\
Total & 65 & 195 & & \\
\hline
\end{tabular}

D. immitis= Dirofilaria immitis 
Table-4: Association of hematological parameters and $D$. immitis infection in pet and semi-domesticated cats.

\begin{tabular}{lccc}
\hline $\begin{array}{l}\text { Hematological } \\
\text { results }\end{array}$ & \multicolumn{2}{c}{ D. immitis infection } & p-value \\
\cline { 2 - 3 } & Positive (n) & Negative (n) & \\
\hline Anemia & 3 & 16 & 0.718 \\
Yes & 26 & 182 & \\
$\quad$ No & & 30 & 0.278 \\
Leukocytosis & 7 & 168 & \\
Yes & 22 & & \\
No & & 92 & 0.045 \\
Eosinophilia & 20 & 101 & \\
Yes & 9 & & \\
No & 9 &
\end{tabular}

D. immitis=Dirofilaria immitis

Table-5: Association of hematological parameters and Wolbachia infection in pet and semi-domesticated cats.

\begin{tabular}{lccc}
\hline $\begin{array}{l}\text { Hematologic } \\
\text { results }\end{array}$ & \multicolumn{2}{c}{ Wolbachia infection } & p-value \\
\cline { 2 - 3 } & $\begin{array}{c}\text { Positive } \\
\text { (n) }\end{array}$ & $\begin{array}{c}\text { Negative } \\
\text { (n) }\end{array}$ & \\
\hline $\begin{array}{l}\text { Anemia } \\
\text { Yes }\end{array}$ & 4 & 15 & 0.764 \\
$\quad$ No & 39 & 169 & \\
$\begin{array}{l}\text { Leukocytosis } \\
\text { Yes }\end{array}$ & 9 & 28 & 0.364 \\
No & 34 & 156 & \\
$\begin{array}{l}\text { Eosinophilia } \\
\text { Yes }\end{array}$ & 27 & 85 & 0.089 \\
No & 16 & 94 & \\
\hline
\end{tabular}

are usually infected with a low number of mature heartworms; moreover, worms exhibit a short lifespan, and only female heartworms are detectable by the antigen test [20]. In the present study, PCR detection of microfilaria was used to identify heartworm infection. A PCR-based method provides highly sensitive and specific detection of heartworm infection, and this method has been demonstrated to identify occult heartworm infections in dogs [21]. Our PCR-based assay results suggest a higher prevalence of heartworm infection in semi-domesticated cats than in pet cats. These findings make sense, since semi-domesticated cats reside with dogs and live outdoors.

Although cats are atypical hosts for heartworms, our findings indicated a high prevalence of semi-domesticated cats positive for heartworms (27.7\%). A lower prevalence of heartworm infection among pet cats $(3.9 \%)$ was detected in this study. The discrepancy between the two cat populations may be because pet cats regularly receive anthelmintic medications, which may be combined with flea prevention products. Most pet cats live indoors with humans and are thus protected from mosquito bites. Moreover, semi-domesticated cats share their habitats with dogs, which are a major reservoir of $D$. immitis.

Doxycycline is recommended for dogs with heartworm infection to treat concurrent Wolbachia endosymbiont infection $[13,22]$. This study demonstrated a strong relationship between $D$. immitis and Wolbachia infections $(\mathrm{p}<0.001)$. Thus, antimicrobial drugs for the treatment of Wolbachia infection should be considered along with anthelmintic drugs to treat heartworm infection in cats and dogs. The number of cats with positive $D$. immitis infection $(\mathrm{n}=51)$ was lower than those with positive Wolbachia infection $(\mathrm{n}=65)$. All cats positive for D. immitis were also positive for Wolbachia infection. This may be because the Wolbachia genus is an obligate intracellular bacteria identified in many nematodes such as Dirofilaria spp. and Brugia spp. [13,15]. Wolbachia infection identified in our study may be acquired from $D$. immitis and other nematode species. Moreover, a study recently reported $B$. malayi and Wolbachia endosymbiont infection in cats residing in Thailand [15]. It is possible that $B$. malayi may be a contributing factor to the result of cats being negative for D. immitis but positive for Wolbachia infection.

The hematological findings indicated that positive D. immitis infection is associated with the presence of eosinophilia. Eosinophils are an important contributor to eliminating nematode infestations through a type 2 immune response [23-25]. Heartworm disease in cats can also lead to the development of eosinophilic pneumonitis [17]. The release of the Wolbachia from filarial nematodes may elicit a host immune response by activating eosinophils, leading to the release of granule proteins. Moreover, the presence of anemia and leukocytosis was not associated with $D$. immitis or Wolbachia endosymbiont infection, which may be because the enrolled cats in this study were clinically healthy at the time of sample collection. Thus, this study found no relationship between abnormal hematological profiles (except eosinophilia) and infection with D. immitis or Wolbachia.

\section{Conclusion}

From the PCR analysis, it can be concluded that there was a significant association between positive D. immitis infection and positive Wolbachia infection. Semi-domesticated cats were at higher risk from $D$. immitis and Wolbachia infection than pet cats. As our study was based on small sample size, further studies with larger sample size should be performed to determine the relationship between hematological abnormalities in cats with either D. immitis or Wolbachia infection. Non-antimicrobial compounds, such as antioxidants and antihistamines, may also prove useful to minimize the host immune response to filarial infection.

\section{Authors' Contributions}

NT and TI: Designed the study, performed the experimental work, and reviewed the manuscript. SA: Performed the study and reviewed the manuscript. JMS: Data interpretation, project advisor, and reviewed the manuscript. PS: Designed the study, conducted the literature review, performed the experimental work, interpreted the data, and drafted the manuscript. All authors read and approved the final manuscript.

\section{Acknowledgments}

The authors would like to thank the animal owners for their cooperation and the staff at the Kasetsart 
University Veterinary Teaching Hospital, Bangkhen Campus. This study was supported by a grant from the Faculty of Veterinary Medicine, Kasetsart University (Grant no. \#62 04), and by the Office of the Ministry of Higher Education, Science, Research and Innovation and the Thailand Science Research and Innovation through the Kasetsart University Reinventing Program 2021 to Panpicha Sattasathuchana.

\section{Competing Interests} interests.

The authors declare that they have no competing

\section{Publisher's Note}

Veterinary World remains neutral with regard to jurisdictional claims in published institutional affiliation.

\section{References}

1. Al-Abd, N.M., Nor, Z.M., Kassim, M., Mansor, M., Al-Adhroey, A.H., Ngui, R. and Sivanandam, S. (2015) Prevalence of filarial parasites in domestic and stray cats in Selangor State, Malaysia. Asian. Pac. J. Trop. Med., 8(9): 705-709.

2. Lu, T.L., Wong, J.Y., Tan, T.L. and Hung, Y.W. (2017) Prevalence and epidemiology of canine and feline heartworm infection in Taiwan. Parasit. Vectors., 10(Suppl 2): 484.

3. Kamyingkird, K., Junsiri, W., Chimnoi, W., Kengradomkij, C., Saengow, S., Sangchuto, K., Kajeerum, W., Pangjai, D., Nimsuphan, B., Inpankeaw, T. and Jittapalapong, S. (2017) Prevalence and risk factors associated with Dirofilaria immitis infection in dogs and cats in Songkhla and Satun Provinces, Thailand. Agric. Nat. Resour., 51(4): 299-302.

4. Levy, J.K., Snyder, P.S., Taveres, L.M., Hooks, J.L., Pegelow, M.J., Slater, M.R., Hughes, K.L. and Salute, M.E. (2003) Prevalence and risk factors for heartworm infection in cats from Northern Florida. J. Am. Anim. Hosp. Assoc., 39(6): 533-537.

5. Nuchprayoon, S., Junpee, A., Nithiuthai, S., Chungpivat, S., Suvannadabba, S. and Poovorawan, Y. (2006) Detection of filarial parasites in domestic cats by PCR-RFLP of ITS1. Vet. Parasitol., 140(3-4): 366-372.

6. Sukhumavasi, W., Bellosa, M.L., Lucio-Forster, A., Liotta, J.L., Lee, A.C., Pornmingmas, P., Chungpivat, S., Mohammed, H.O., Lorentzen, L., Dubey, J.P. and Bowman, D.D. (2012) Serological survey of Toxoplasma gondii, Dirofilaria immitis, Feline Immunodeficiency Virus (FIV) and Feline Leukemia Virus (FeLV) infections in pet cats in Bangkok and vicinities, Thailand. Vet. Parasitol., 188(1-2): 25-30.

7. Ciuca, L., Simon, F., Rinaldi, L., Kramer, L., Genchi, M., Cringoli, G., Acatrinei, D., Miron, L. and Morchon, R. (2018) Seroepidemiological survey of human exposure to Dirofilaria spp. in Romania and Moldova. Acta. Trop., 187: 169-174.

8. Darby, B., Dickinson, C., Gaskins, L. and Hanna, P. (2019) Prevalence of heartworm infection in the feral cat population of Grand Cayman. JFMS Open. Rep., 5(1): 2055116919838083.

9. Snyder, P.S., Levy, J.K., Salute, M.E., Gorman, S.P., Kubilis, P.S., Smail, P.W. and George, L.L. (2000) Performance of serologic tests used to detect heartworm infection in cats. J. Am. Vet. Med. Assoc., 216(5): 693-700.

10. Carretón, E., Morchón, R. and Montoya-Alonso, J.A. (2017) Cardiopulmonary and inflammatory biomarkers in heartworm disease. Parasit. Vectors., 10(Suppl 2): 534

11. Nelson, C.T., Myrick, E.S. and Nelson, T.A. (2017) Clinical benefits of incorporating doxycycline into a canine heartworm treatment protocol. Parasit. Vectors., 10(Suppl 2): 515.

12. Dingman, P., Levy, J.K., Kramer, L.H., Johnson, C.M., Lappin, M.R., Greiner, E.C., Courtney, C.H., Tucker, S.J. and Morchon, R. (2010) Association of Wolbachia with heartworm disease in cats and dogs. Vet. Parasitol., 170(1-2): 50-60.

13. Kramer, L.H., Passeri, B., Corona, S., Simoncini, L. and Casiraghi, M. (2003) Immunohistochemical/immunogold detection and distribution of the endosymbiont Wolbachia of Dirofilaria immitis and Brugia pahangi using a polyclonal antiserum raised against WSP (Wolbachia surface protein). Parasitol. Res., 89(5): 381-386.

14. Taylor, M.J., Cross, H.F. and Bilo, K. (2000) Inflammatory responses induced by the filarial nematode Brugia malayi are mediated by lipopolysaccharide-like activity from endosymbiotic Wolbachia bacteria. J. Exp. Med., 191(8): 1429-1436.

15. Khowawisetsut, L., Sarasombath, P.T., Thammapalo, S., Loymek, S., Korbarsa, T., Nochote, H., Phuakrod, A., Choochet, W. and Wongkamchai, S. (2017) Therapeutic trial of doxycyclin plus ivermectin for the treatment of Brugia malayi naturally infected cats. Vet. Parasitol., 245: 42-47.

16. Satjawongvanit, H., Phumee, A., Tiawsirisup, S., Sungpradit, S., Brownell, N., Siriyasatien, P. and Preativatanyou, K. (2019) Molecular analysis of canine filaria and its Wolbachia endosymbionts in domestic dogs collected from two animal university hospitals in Bangkok metropolitan region, Thailand. Pathogens, 8(3): 114.

17. Turba, M.E., Zambon, E., Zannoni, A., Russo, S. and Gentilini, F. (2012) Detection of Wolbachia DNA in blood for diagnosing filaria-associated syndromes in cats. J. Clin. Microbiol. 50(8): 2624-2630.

18. Slatko, B.E., Luck, A.N., Dobson, S.L. and Foster, J.M. (2014) Wolbachia endosymbionts and human disease control. Mol. Biochem. Parasitol., 195(2): 88-95.

19. Sabūnas, V., Radzijevskaja, J., Sakalauskas, P., Petkevičius, S., Karvelienè, B., Žiliukienè, J., Lipatova, I. and Paulauskas, A. (2019) Diroflaria repens in dogs and humans in Lithuania. Parasit. Vectors, 12(1): 177.

20. Rishniw, M., Barr, S.C., Simpson, K.W., Frongillo, M.F., Franz, M. and Dominguez Alpizar, J.L. (2006) Discrimination between six species of canine microfilariae by a single polymerase chain reaction. Vet. Parasitol., 135(3-4), 303-314.

21. Little, S.E., Raymond, M.R., Thomas, J.E., Gruntmeir, J., Hostetler, J.A., Meinkoth, J.H. and Blagburn, B.L. (2014) Heat treatment prior to testing allows detection of antigen of Dirofilaria immitis in feline serum. Parasit. Vectors, 7: 1.

22. Trancoso, T.A.L., Lima, N.D.C., Barbosa, A.S., Leles, D., Fonseca, A.B.M., Labarthe, N.V., Bastos, O.M.P. and Uchôa, C.M.A. (2020) Detection of Dirofilaria immitis using microscopic, serological and molecular techniques among dogs in Cabo Frio, RJ, Brazil. Rev. Bras. Parasitol. Vet., 29(1): e017219.

23. Kramer, L.H., Crosara S., Gnudi G., Genchi M., Mangia C., Viglietti A. and Quintavalla C. (2018) Wolbachia, doxycycline and macrocyclic lactones: New prospects in the treatment of canine heartworm disease. Vet. Parasitol., 254: 95-97.

24. Anthony, R.M., Rutitzky L.I., Urban Jr, J.F., Stadecker, M.J. and Gause, W.C. (2007) Protective immune mechanism in helminth infection. Nat. Rev. Immunol., 7(12): 975-987.

25. Sattasathuchana, P. and Steiner, JM. (2014) Canine eosinophilic gastrointestinal disorders. Anim. Health. Res. Rev., 15(1): 76-86. 